\title{
A Study on the Relationship between the Score of Teachers and the Ratio of Grade A
}

\author{
Jianjun Yin \\ School of information science and technology \\ Fudan University \\ Shanghai, China \\ yinjianjun@fudan.edu.cn
}

\begin{abstract}
This article made a research on the relationship between the score of the teacher and the students' grade of the school of information science and technology of Fudan University. Firstly, we selected the samples, which came from all the courses those had more than 10 valid questionnaires (including 10). Secondly, we divided the score of teachers into several paragraphs. Then the proportion of $A$ (including A-) in each segmentation was analyzed. Through the data analysis of several semesters, we draw the following conclusion: each term is slightly different, in general, there is no necessary relationship between the score of teachers given by students and the grade of $A$ given by teachers.
\end{abstract}

Keywords-Education, Teacher score, Ratio of grade A, Relationship

\section{INTRODUCTION}

For some speculation, each semester teachers' score given by students is relative with the students' grades given by the teachers. That is, those give high grades to students may get relatively high score. We launched a targeted research, i.e., the relationship between the score and grade A (including A-) ratio. This study used data from four semesters: 2012-2013 second semester, 2013-2014 first semester, 2013-2014 second semester and 2014-2015 first semester [1-6]. In the paper all the score or grades used are average values. The score refers to the teachers' score given by the students and the grades refer to the students' final achievements given by the teachers. As the situation is not the same, we will discuss the situation based on each semester. We divided the score of teachers into several paragraphs. Then the proportion of A (including A-) in each segmentation was analyzed [7]. The following chapters are organized as follows. Part 2 is the analysis for the dada of the 2012-2013 second semester; Part 3 is the analysis for the dada of 2013-2014 first semester; Part 4 is the analysis for the dada of the 2013-2014 second semester; Part 5 is the 2014-2015 first semester data analysis. Finally, it is a summary of the thesis.

\section{THE SECOND SEMESTER IN 2012-2013}

\section{A. Sample selection}

The data came from all the courses those had more than 10 valid questionnaires (including 10), including a total of 82 courses $[1,2]$.

\section{B. Description}

In this semester, teacher ratings are generally high, in accordance with the requirements of the sample, all the teacher score are 4 points above(the full score is 5 ), the distribution is shown in Fig. 1 below, where we have 41 courses ( 8 of them got a full mark) in the score segmentation [4.9, 5], and 24 courses in segmentation [4.8, 4.9), 9 courses in segmentation $[4.7,4.8), 8$ courses in segmentation $[4.0,4.7)$.

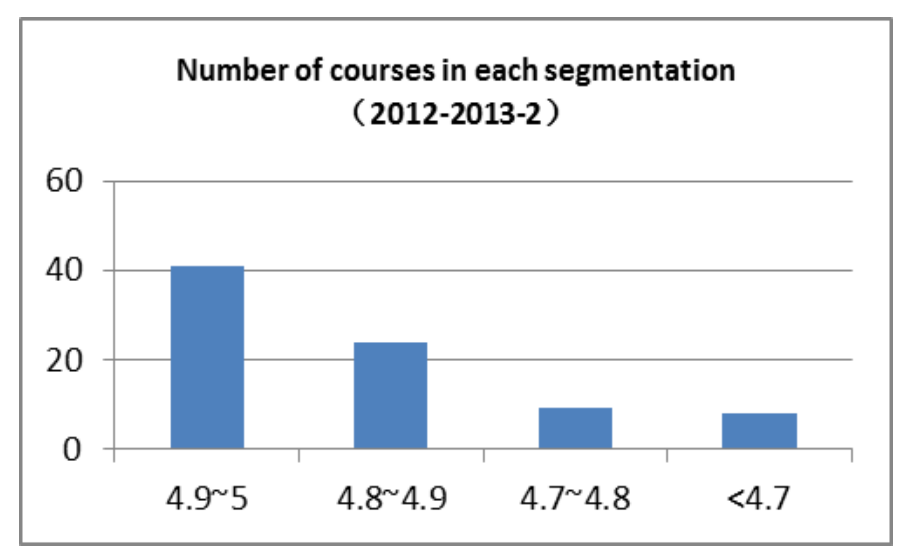

Fig. 1. 2012-2013-2 Number of courses in each segmentation.

\section{Analysis}

Fig. 2 shows the proportion of A (including A-) in each segmentation. We can see from Fig. 2 that the average proportion of A of segmentation $[4.9,5]$ and $[4.8,4.9)$ is about $28 \%$, which is slightly higher than that of segmentation [4.7, 4.8,) and [4.0, 4.7), which is approximately 25\%. And we can make a preliminary conclusion that there is a slight connection between the teachers' score given by the students and the students' grade A given by the teachers. 


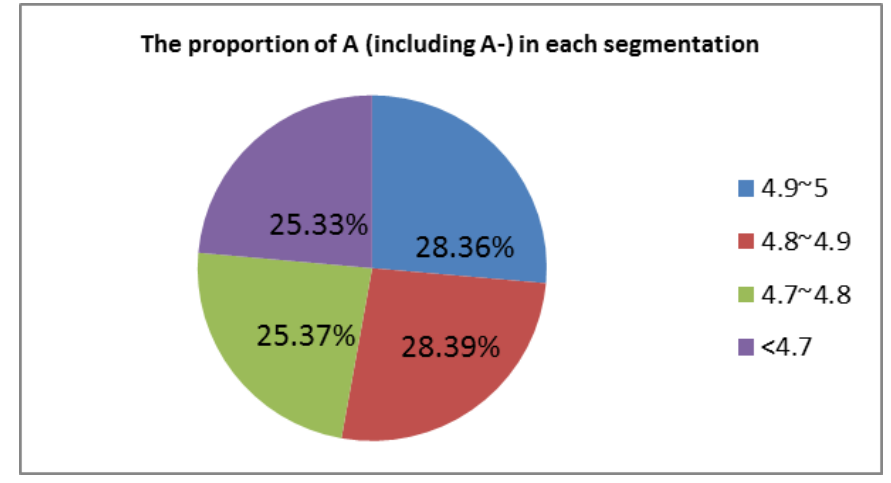

Fig. 2. 2012-2013-2 The proportion of A (including A-) in each segmentation.

\section{THE FIRST SEMESTER IN 2013-2014}

\section{A. Sample selection}

The data came from all the courses those had more than 10 valid questionnaires (including 10), including a total of 94 courses [3, 4].

\section{B. Description}

In accordance with the requirements of the sample, all the teacher score are 3.9 points above (the full score is 5), the distribution is shown in Fig. 3 below, where we have 6 courses ( 1 of them got a full mark) in the score segmentation [4.9, 5], and 15 courses in segmentation [4.8, 4.9), 21 courses in segmentation $[4.7,4.8), 19$ courses in segmentation [4.6, 4.7), 9 courses in segmentation [4.5, 4.6), 9 courses in segmentation $[4.4,4.5), 7$ courses in segmentation $[4.2,4.4), 8$ courses in segmentation [3.9, 4.2).

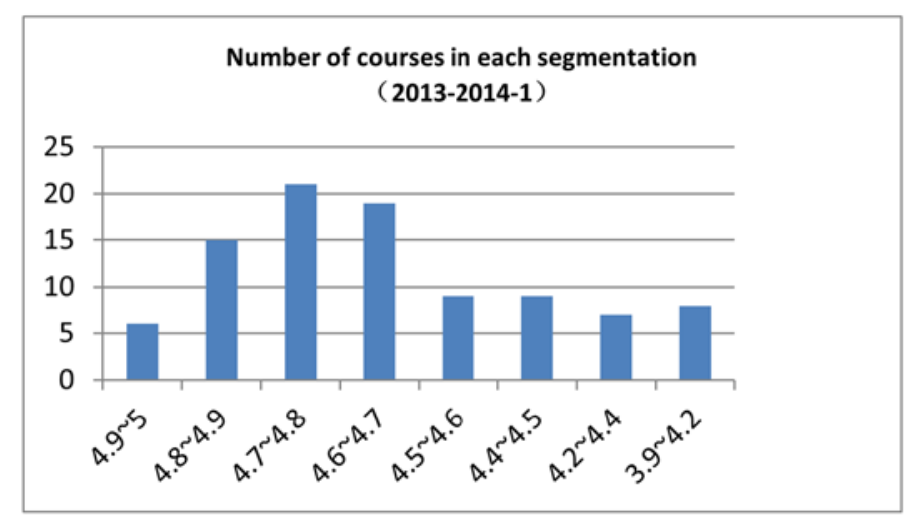

Fig. 3. 2013-2014-1 Number of courses in each segmentation.

\section{Analysis}

Fig. 4 shows the proportion of A (including A-) in each segmentation. We can see from Fig. 4that the second part of the histogram is slightly lower than the first half scores. And we can make a preliminary conclusion that there is a slight connection between the teachers' score given by the students and the students' grade A given by the teachers. That is the teachers who give more as to students may likely to get high marks.

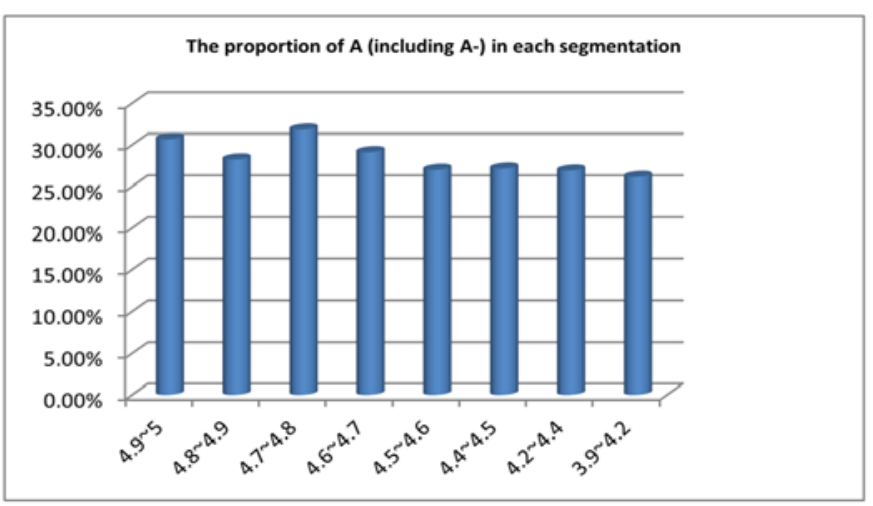

Fig. 4. 2013-2014-1 The proportion of A (including A-) in each segmentation.

\section{THE SECOND SEMESTER IN 2013-2014}

\section{A. Sample selection}

The data came from all the courses those had more than 10 valid questionnaires (including 10), including a total of 91 courses $[3,4]$.

\section{B. Description}

In accordance with the requirements of the sample, all the teacher score are 3.5 points above (the full score is 5), the distribution is shown in Fig. 5 below, where we have 8 courses (1 of them got a full mark) in the score segmentation [4.9, 5], and 11 courses in segmentation [4.8, 4.9), 17 courses in segmentation $[4.7,4.8), 13$ courses in segmentation $[4.6,4.7)$, 16 courses in segmentation $[4.5,4.6), 9$ courses in segmentation [4.4, 4.5), 10 courses in segmentation [4.2, 4.4), 7 courses in segmentation [3.5, 4.2).

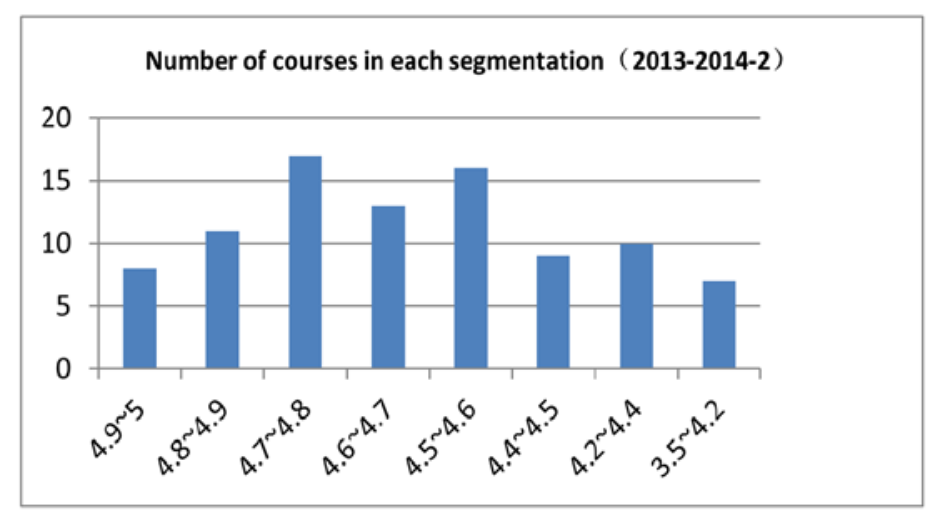

Fig. 5. 2013-2014-2 Number of courses in each segmentation.

\section{Analysis}

Fig. 6 shows the proportion of A (including A-) in each segmentation. We can see from Fig. 6 that histogram is nonmonotonic. And we can make a preliminary conclusion that there is no connection between the teachers' score given by the students and the students' grade A given by the teachers. 


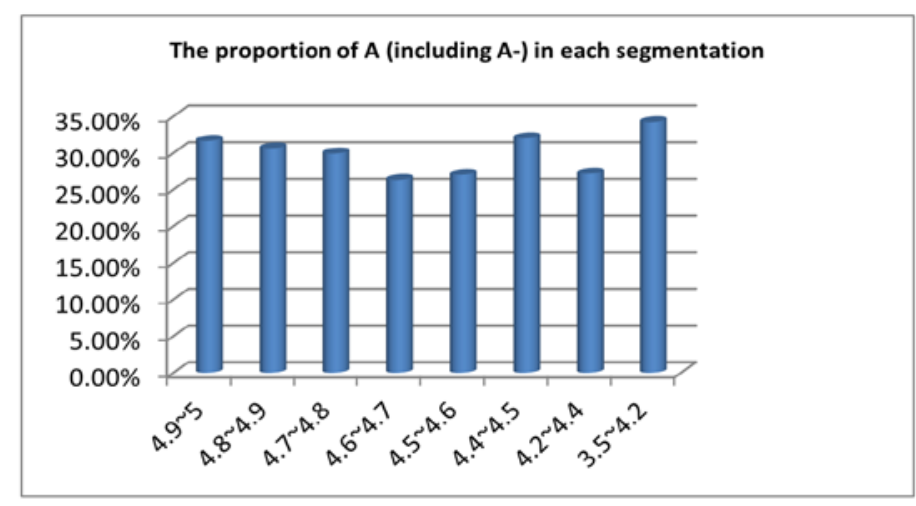

Fig. 6. 2013-2014-2 The proportion of A (including A-) in each segmentation.

\section{THE FIRST SEMESTER IN 2014-2015}

\section{A. Sample selection}

The data came from all the courses those had more than 10 valid questionnaires (including 10), including a total of 77 courses $[5,6]$.

\section{B. Description}

In accordance with the requirements of the sample, all the teacher score are 3.9 points above (the full score is 5), the distribution is shown in Fig. 7 below, where we have 8 courses ( 0 of them got a full mark) in the score segmentation $[4.9,5]$, and 10 courses in segmentation [4.8, 4.9), 12 courses in segmentation $[4.7,4.8), 14$ courses in segmentation [4.6, 4.7), 16 courses in segmentation $[4.5,4.6), 4$ courses in segmentation [4.4, 4.5), 9 courses in segmentation [4.2, 4.4) , 4 courses in segmentation [3.9, 4.1).

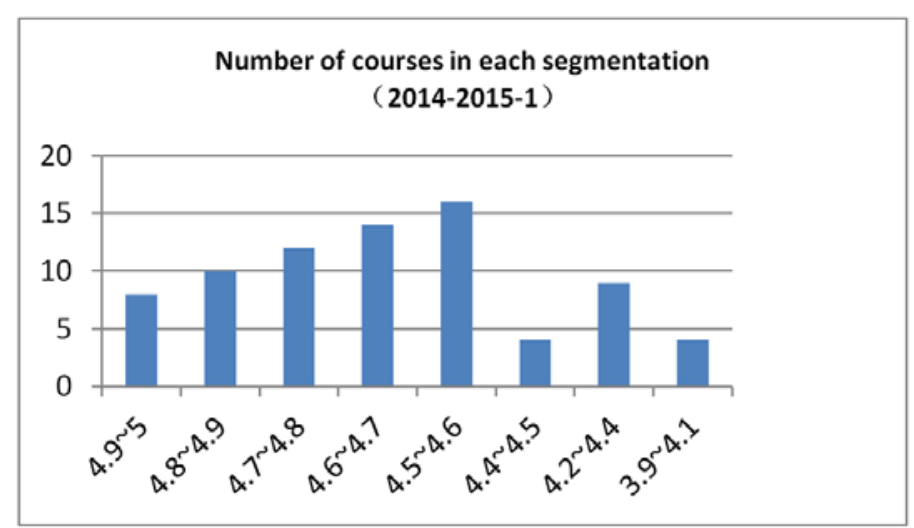

Fig. 7. 2014-2015-1 NumBer of courses in each segmentation.

\section{Analysis}

Fig. 8 shows the proportion of A (including A-) in each segmentation. We can see from Fig. 8 that histogram is nonmonotonic. And we can also make a preliminary conclusion that there is no obvious connection between the teachers' score given by the students and the students' grade A given by the teachers.

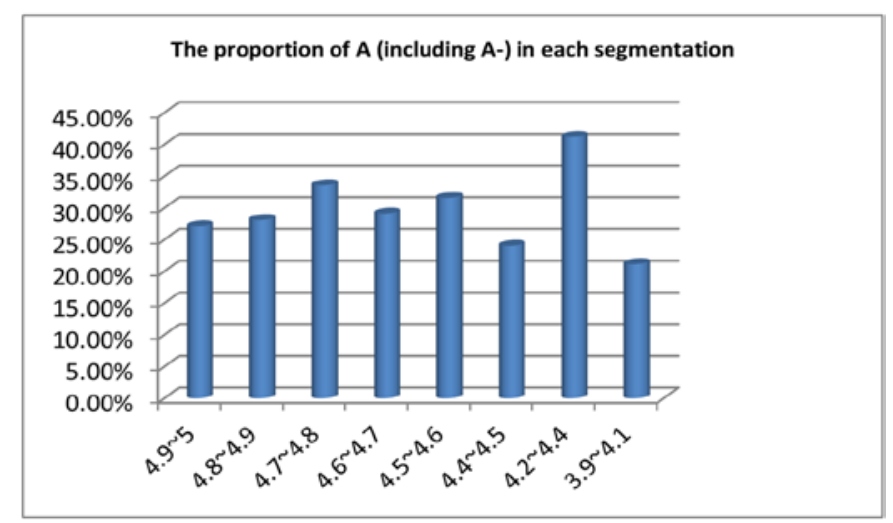

Fig. 8. 2014-2015-1 The proportion of A (including A-) in each segmentation.

\section{SUMMARY}

This paper makes a preliminary analysis of the relations between the score of teachers and the ratios of grade $\mathrm{A}$ for students, from the samples and analysis of the above four semesters, each semester are different. In two semesters, the analysis results showed slight contact between them, while in the other two semesters, there is no obvious contact.

\section{REFERENCES}

[1] The Student achievements of the second semester in 2012-2013. School of information science and technology, Fudan University.

[2] The teaching score of the second semester in 2012-2013. School of information science and technology, Fudan University.

[3] The Student achievements of the first and second semester in 2013-2014. School of information science and technology, Fudan University.

[4] The teaching score of the first and second semester in 2013-2014. School of information science and technology, Fudan University.

[5] The Student achievements of the first semester in 2014-2015. School of information science and technology, Fudan University.

[6] The teaching score of the first semester in 2014-2015. School of information science and technology, Fudan University.

[7] Wang Deqin, Excel spreadsheet software in the construction of the teaching staff-some applications, Journal of Changzhi University, vol. 31, pp. 57-59, 2014 